\title{
Combination Chemotherapy of Mitomycin C and Methotrexate Was Effective on Metastatic Breast Cancer Resistant to Eribulin, Vinorelbine, and Bevacizumab after Anthracycline, Taxane, and Capecitabine
}

\author{
Masahiko Tanabe \\ Department of Breast Surgical Oncology, Kyoundo Hospital, Tokyo, Japan; Department of \\ Breast Surgery and Oncology, Juntendo University, Tokyo, Japan
}

\section{Keywords}

Metastatic breast cancer - Mitomycin C - Methotrexate

\begin{abstract}
Complete cure of metastatic breast cancer (MBC) is still considered difficult even after the development of new drugs. While new drugs have been continuously developed, conventional drugs such as mitomycin $C$ (MMC) and methotrexate (MTX) have become less used. Combination chemotherapy with MMC and MTX (MMC/MTX) was reported to be effective for $9.7-19.4 \%$ of 31 patients with human epidermal growth factor receptor type 2 (HER2)negative MBC who were aggressively treated with anthracycline, taxane, capecitabine, and vinorelbine. However, its efficacy, when it is used after newly developed drugs such as eribulin and bevacizumab, is yet to be evaluated. We here introduce one case in which MMC/MTX was effective for $M B C$ that was resistant to chemotherapy with eribulin, vinorelbine, and bevacizumab with paclitaxel after sequential treatment with anthracycline, taxane, capecitabine, and several hormonal therapies. Lung metastasis was newly observed after sequential
\end{abstract}


Tanabe: Mitomycin C and Methotrexate for Metastatic Breast Cancer Refractory to New Drugs

treatment of MBC for 6 years. Although the disease was resistant to chemotherapy of eribulin, vinorelbine, and bevacizumab with paclitaxel, it responded well to the treatment of MMC/MTX, which continued for 7 months. This case suggests that MMC/MTX could be an effective treatment for $M B C$ patients when the disease progressively develops even after aggressive treatment with multiple regimens.

(C) 2016 The Author(s)

Published by S. Karger AG, Basel

\section{Background}

Complete cure of metastatic breast cancer (MBC) is still considered difficult even after the development of new drugs. Patients with MBC, who have kept a good performance status (PS), are eager for the next effective treatment to keep their MBC under control and to maintain their quality of life even after all standard treatments according to the guidelines have been administered. Therefore, it is important for physicians to have several alternative treatments for such MBC patients. While new drugs have been continuously developed, conventional drugs such as mitomycin C (MMC) and methotrexate (MTX), which are well documented for efficacy and safety, have become less used. Combination chemotherapy with MMC and MTX (MMC/MTX) was reported to be effective for $24 \%$ of 48 patients whose MBC had been treated with anthracycline and taxane, and in which the median time to progression was 4.8 months [1]. In addition, MMC/MTX was reported to be effective for 9.7-19.4\% of 31 patients with human epidermal growth factor receptor type 2 (HER2)-negative MBC who were aggressively treated with anthracycline, taxane, capecitabine, and vinorelbine, and in which the median time to progression was 3.9 months [2]. We consider that this MMC/MTX treatment would have the possibility to be one choice of treatment for MBC patients when they keep a good PS even after heavy treatment. However, its efficacy, when it is used after newly developed drugs such as eribulin and bevacizumab, is yet to be evaluated.

\section{Case Presentation}

A woman who had breast cancer with metastases in several lymph nodes and multiple bones was diagnosed as MBC (T4N3M1, stage IV). The primary tumor in her breast was invasive ductal carcinoma, and immunohistochemistry of the tumor revealed that it was positive for both estrogen and progesterone receptor and negative for HER2. First treatment of the disease was initiated with an anthracycline-containing regimen of FEC (5FU, epirubicin, and cyclophosphamide) for 4 months followed by taxane (weekly paclitaxel) for 12 months. After treatment with anthracycline and taxane, the tumor of the breast and lymph nodes in the axilla became smaller to the point of not being measurable. On bone metastases, initially bisphosphonate and afterwards denosumab were administered. Precise evaluation of the disease in the bone was difficult from the beginning. Thereafter, we evaluated the efficacy of treatment by use of the tumor markers carcinoembryonic antigen (CEA) and cancer antigen 15-3 (CA15-3), as shown in figure 1. When the tumor markers elevated, the treatment was changed to the next one sequentially, as mentioned below. After anthracycline and taxane, hormonal treatments with tamoxifen for 8 months, letrozole for 10 months, toremifene for 4 months, and exemestane for 3 months were continued. When exemestane could not control the elevation of the tumor markers, treatment was changed to capecitabine. Though capecitabine had controlled the disease for 12 months, liver metastases were newly observed. Three months after treatment of fulvestrant, a lung metastasis was newly observed. At this 


\section{Case Reports in Oncology}

time, it had been 6 years since the initial treatment. Although chemotherapy was restarted against the progressive MBC, treatment with eribulin for 3 months, vinorelbine for 2 months and bevacizumab with paclitaxel for 2 months had no power to suppress the continuous progression of the disease. The size of the lung metastasis became more than $5 \mathrm{~cm}$ in diameter and the tumor markers of CEA and CA15-3 had continuously elevated, as shown in figure 2. While the MBC progressed continuously despite any treatment, her PS and function of bone marrow were good even after sequential multiple regimens. She expected the next chemotherapy that was not previously administered. In this situation, gemcitabine, MMC/ MTX, and everolimus with exemestane were alternatives of treatment. Because the disease had continuously progressed, a regimen with which rapid response would be obtained was expected as the next treatment. Considering the potential response rate and adverse effect of treatment, MMC/MTX was selected. One cycle of MMC/MTX was defined as MMC $8 \mathrm{mg} / \mathrm{m}^{2}$ on day 1 and MTX $60 \mathrm{mg} / \mathrm{m}^{2}$ on days 1 and 15, administered intravenously every 4 weeks, as previously reported $[1,2]$. This regimen has been admitted and registered by the review board of chemotherapy. Written informed consent was obtained before this treatment was started. Firstly, three cycles of this treatment were completed as scheduled. In this sequential course of MMC/MTX treatment, the continuously elevating tumor markers decreased for the first time after the disease showed resistance to any chemotherapy (fig. 2). Hematuria (grade one, G1, in the Common Terminology Criteria for Adverse Events, version 3.0) was observed after three cycles, which spontaneously improved with no treatment. Interstitial pneumonia (G2) was observed after 4 cycles, which needed a skip of treatment as mentioned below. MTX on day 15 in the fourth course was skipped. Oral steroid was medicated on this interstitial pneumonia. In the fifth course, only MTX without MMC was administered on day 1 and day 15 until improvement of the interstitial pneumonia was confirmed. Neutropenia (G4), decrease of platelet counts (G3), and anemia (G3) were observed after five cycles, which needed postponement of the start date of the next cycle for 2 weeks. In the sixth course, MMC was re-administered on day 1, and MMC/MTX treatment was completed as scheduled. At this point, the cumulative dose of MMC reached to $40 \mathrm{mg} / \mathrm{m}^{2}$ administered five times in six cycles. Considering hematological toxicity, the dose of MMC was reduced to $50 \%$ $\left(4 \mathrm{mg} / \mathrm{m}^{2}\right)$ in the seventh and eighth courses, which were completed as scheduled. The MMC/MTX treatment had finally controlled this disease for 7 months until progressive enlargement of the lung metastasis was observed.

This is only one case shown here; however, MMC/MTX was able to control progression of the disease for 7 months even though it was difficult to control the disease with newly developed drugs as mentioned. It is speculated that the reason why MMC/MTX was effective in this case is that pharmacological actions of MMC/MTX would be different from those of previously used anticancer drugs. MMC functions by forming cross-links in DNA in a cell cycle-nonspecific manner [3]. MTX inhibits dehydrofolate reductase, which inhibits DNA synthesis in a cell cycle-specific manner [4]. The efficacy of MMC/MTX would be attributed to no cross-resistance with previous treatment. In addition, a synergistic effect of MMC and MTX was reported [5-7].

In which case is MMC/MTX beneficial for MBC patients? I recommend this treatment for MBC patients who satisfy the following conditions: (1) PS and bone marrow function are good. (2) MBC is resistant to other multiple treatments according to the guidelines. (3) Previous regimens do not include drugs with a similar function of MMC and MTX. Because myelosuppression might become a problem later when MMC is administered in an earlier line, this treatment is recommended as an alternative option not in an earlier line but in a later line. It is very difficult to conduct clinical studies including randomized controlled ones on 
the treatment of MBC in a late line after multiple treatments. Therefore, we assume that case reports like this would be very significant when we are going to do something that is best for MBC patients to keep them in a similar condition as long as possible.

\section{Conclusion}

This case suggests that MMC/MTX could be an effective treatment for patients whose MBC progressively develops even after aggressive treatment with multiple regimens. It can be presumed that the effectiveness of MMC/MTX lies in their pharmacological mechanisms that are different from previously used anticancer drugs such as inhibitors of microtubules. We speculate that a further study of MMC/MTX for MBC after heavy treatment would reveal patients who would benefit from MMC/MTX as well as the biology of MBC resistant to multiple regimens of chemotherapy.

\section{Acknowledgements}

This case was reported at the World Congress on Controversies in Breast Cancer (Melbourne, Australia, October 22-24, 2015).

\section{Statement of Ethics}

The patient in this case report agreed to this. She always hoped to be of some help to MBC patients even after her death. I really respect and appreciate her living will.

\section{Disclosure Statement}

I have no competing interests to declare.

\section{References}

1 Tanabe M, Ito Y, Tokudome N, Sugihara T, Miura H, Takahashi S, Seto Y, Iwase T, Hatake K: Possible use of combination chemotherapy with mitomycin $\mathrm{C}$ and methotrexate for metastatic breast cancer pretreated with anthracycline and taxanes. Breast Cancer 2009;16:301-306.

2 Fukuda T, Tanabe M, Kobayashi K, Fukada I, Takahashi S, Iwase T, Ito Y: Combination chemotherapy with mitomycin $\mathrm{C}$ and methotrexate is active against metastatic HER2-negative breast cancer even after treatment with anthracycline, taxane, capecitabine, and vinorelbine. Springerplus 2015;4:376. Hortobagyi GN: Mitomycin-C in breast cancer. Semin Oncol 1985;12(4 suppl 6):65-70.

Chabner BA, Roberts TG Jr: Timeline: chemotherapy and the war on cancer. Nat Rev Cancer 2005;5:6572.

5 Mabel JA, Wodinsky I: Mitomycin C combination therapy against murine tumor systems. Effectiveness with cyclophosphamide and methotrexate. Cancer 1983;51:600-605.

6 Jia M, Li Y, Yang X, Huang Y, Wu H, Huang Y, Lin J, Li Y, Hou Z, Zhang Q: Development of both methotrexate and mitomycin C loaded PEGylated chitosan nanoparticles for targeted drug codelivery and synergistic anticancer effect. ACS Appl Mater Interfaces 2014;6:11413-11423.

7 Li Y, Lin J, Wu H, Chang Y, Yuan C, Liu C, Wang S, Hou Z, Dai L: Orthogonally functionalized nanoscale micelles for active targeted codelivery of methotrexate and mitomycin $\mathrm{C}$ with synergistic anticancer effect. Mol Pharm 2015;12:769-782. 


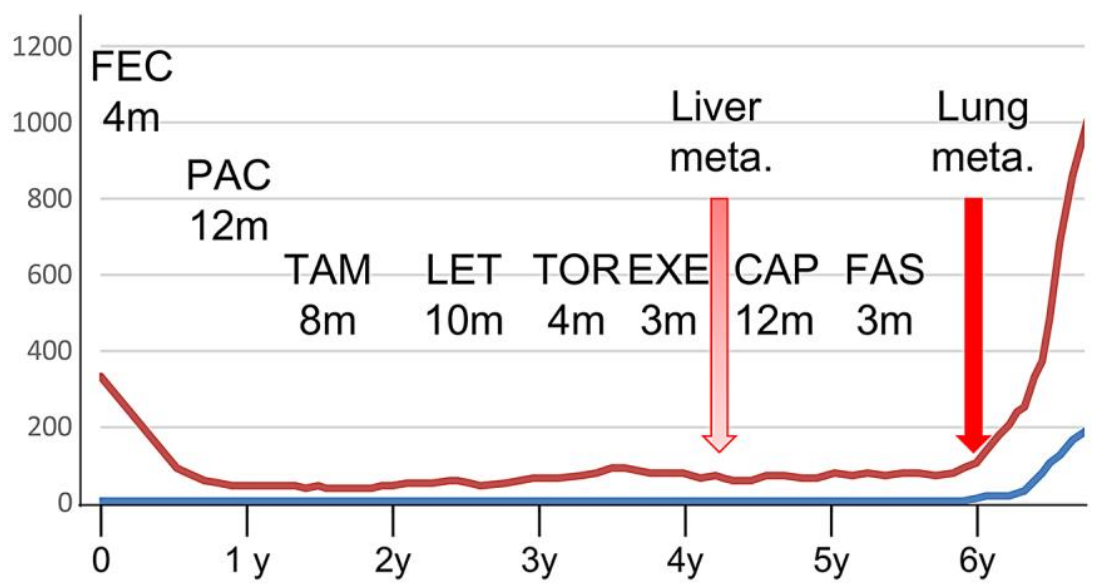

$\longrightarrow$ CEA $\longrightarrow$ CA15-3

Fig. 1. History of previous treatment and change of tumor markers before lung metastasis occurred. FEC = 5FU, epirubicin, and cyclophosphamide; $\mathrm{PAC}=$ weekly paclitaxel; $\mathrm{TAM}$ = tamoxifen; LET = letrozole; TOR = toremifene; $\mathrm{EXE}=$ exemestane; $\mathrm{CAP}=$ capecitabine; $\mathrm{FAS}$ = faslodex; meta. = metastasis $/$ metastases; $\mathrm{y}=$ year(s); $\mathrm{m}=$ months. Values are $\mathrm{ng} / \mathrm{ml}$ for CEA and $\mathrm{U} / \mathrm{ml}$ for CA15-3.

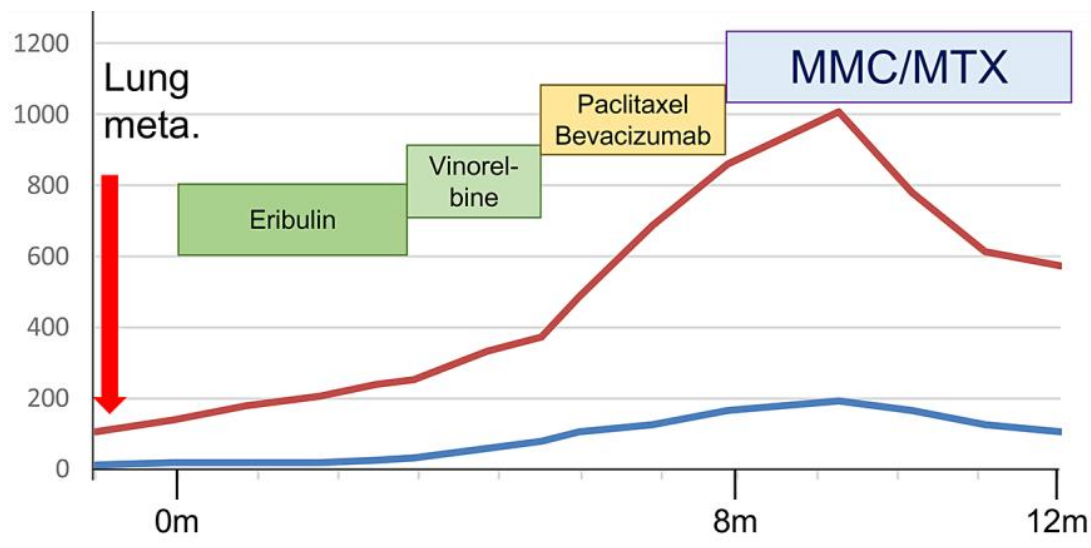

Fig. 2. Treatment history and change of tumor markers after lung metastasis occurred. $m=$ Months. Values are $\mathrm{ng} / \mathrm{ml}$ for CEA and $\mathrm{U} / \mathrm{ml}$ for CA15-3. 